\title{
Células de Estudo de Programação nos Cursos de Engenharia da Universidade Federal do Ceará no Campus Sobral com Aplicação de Metodologia de Ensino Cooperativo
}

\author{
Manoel E. N. Oliveira ${ }^{1}$,Felipe Barros Muniz ${ }^{2}$, Ruann C. Farrapo ${ }^{3}$ \\ ${ }^{1}$ Departamento de Engenharia Elétrica - Universidade Federal do Ceará (UFC) \\ CEP 62.010-560 - Centro - Sobral- CE - Brazil \\ ${ }^{2,3}$ Departamento de Engenharia de Computação- Universidade Federal do Ceará (UFC) \\ CEP 62.010-560 - Centro - Sobral- CE - Brazil \\ \{manoeleric59, felipemuniz, ruann. campos 01$\}$ @alu.ufc.br
}

\begin{abstract}
Programming is difficult to understand for its new practitioners, but on the other hand, it is of paramount importance for better logical training and problem solving, quickly and efficiently, being indispensable for engineering students in work with calculations and graphs. In view of the adversities faced, this article aims at the possible applications and forms of teaching, focusing on the modern methodology called cooperative learning, which aims to share knowledge among participants, with the aim of disseminating knowledge of three languages programming in different study cells, Java in Android development, Python in Machine Learning with Scikit-learn and $C$ in microcontroller applications.
\end{abstract}

Resumo: A programação é de difícil entendimento para os seus novos praticantes, mas por outro lado, é de suma importância para uma melhor capacitação lógica e de resolução de problemas, de forma rápida e eficiente, sendo indispensáveis para estudantes de engenharia em trabalhos com cálculos e gráficos. Em consideração as adversidades enfrentadas, o presente artigo visa as possíveis aplicações e formas de ensino, tendo em foco a metodologia moderna chamada de aprendizagem cooperativa, que visa o compartilhamento de conhecimento entre os participantes, com o intuito da disseminação do conhecimento de três linguagens de programação em células de estudos distintas, sendo Java em desenvolvimento Android, Python em Machine Learning com Scikit-learn e C em aplicações para microcontroladores.

\section{INTRODUÇÃ̃O}

O Programa de Aprendizagem Cooperativa em Células Estudantis (PACCE) na UFC Campus Sobral era um programa em que são articulados grupos(células) de estudo pelos bolsistas que participam de uma seleção onde demonstram projetos, levando em conta o seu conhecimento prévio no assunto que auxilia os membros dessas células na obtenção de conhecimento dos seus respectivos temas através da aplicação de cinco pilares da Aprendizagem Cooperativa, um método de aprendizagem mais dinâmico em relação à formas tradicionais de ensino em que, no geral, o professor é o detentor e o transmissor da informação e cabe ao aluno o único papel de ser o receptor desse conhecimento [SOUSA, 2017]. Seu término foi decretado no Campus Sobral agora neste ano de 2020.

Em cursos de graduação voltados à tecnologia, nota-se uma maior quantidade de conteúdos teóricos em relação às práticas, ao mesmo tempo que há uma dificuldade em relacionar os dois o que acaba gerando uma desmotivação por parte dos estudantes [GOMES; 
SILVEIRA, 2017]. Dito isso, uma abordagem mais dinâmica, com aplicações práticas, em aulas de cunho majoritariamente teóricos vêm como uma forma de estimular os estudantes [SOUSA, 2017].

Na maioria dos currículos dos cursos de Engenharia há sempre disciplinas de cunho introdutório à Programação Computacional [OLIVEIRA \& ALVES, 2001]. Programação, no geral, é algo de muita utilidade dentro dos campos da engenharia. Este artigo terá como foco o Desenvolvimento Android, a Programação em C e Programação em Python, devido a estes serem os temas estudados nas três Células de Estudo de Programação da UFC Campus Sobral no ano de 2019.

Este artigo visa expor a possibilidade de utilização de Linguagens de Programação em projetos práticos palpáveis para melhor compreensão da programação dentro da engenharia, com a utilização da Aprendizagem Cooperativa no ensino, com o intuito de que os alunos se sintam mais motivados e consequentemente contribuam para a menor taxa de evasão nos cursos.

Neste artigo também é apresentado o estudo de caso do desempenho na disciplina de Programação Computacional do Curso de Engenharia da Computação da Universidade Federal do Ceará Campus Sobral que participaram das células de estudos e dos que não participaram.

\section{FUNDAMENTAÇÃO TEÓRICA}

\subsection{Linguagens de programação}

\subsubsection{Desenvolvimento Android voltado para a engenharia}

Dispositivos Android, devido à sua popularização e à maleabilidade da plataforma, são de grande auxílio em projetos que visam, especialmente, reduzir custos, já que desenvolver aplicativos de monitoramento/controle de algum projeto ao invés de ter que criar todo esse sistema de controle/monitoramento do zero é bem mais barato [OLIVEIRA \& BALBINOT, 2014]. Devido a grande vantagem que um dispositivo Android tem de se comunicar via bluetooth e wireless é possível integrá-lo como dispositivo de controle de um protótipo de uma mão robótica feita com Lego e Arduino por exemplo [HUNOLD et al, 2014]. É possível, também, construir um aplicativo com uma interface simples de gerenciamento de irrigação automatizada para simplificar o trabalho de agricultores sem muita instrução [RODRIGUES et al, 2014]. Ou até mesmo criar, com um aplicativo Android, a interface gŕafica de um sistema de eletrocardiograma portátil [OLIVEIRA \& BALBINOT, 2014].

\subsubsection{A linguagem Python na construção de algoritmos de machine learning com Scikit-learn}

A linguagem Python, atualmente, está crescendo na comunidade científica por sua fácil manipulação e por possuir inúmeros módulos e bibliotecas utilizadas em diversas áreas do conhecimento. Python apresenta inúmeras vantagens na sua utilização, uma delas é sua semântica dinâmica que resulta em tratamento flexível de alguns argumentos.

A aprendizagem de máquina (Machine Learning) consiste em fornecer treinamento a uma máquina a partir de uma boa quantidade de dados relevantes para a realização de uma tarefa e para isso é fundamental a utilização de algoritmos para extrair informações de dados e representá-los com um modelo estatístico matemático [CHOLLET, 2017]. 
Um tipo especial deste tipo de algoritmo são as Redes Neurais Artificiais: um conjunto de modelos de aprendizagem de máquina baseado no sistema nervoso humano. Nesse modelo, as unidades de processamento independentes conectam-se uns aos outros por meio de pesos que são ajustados a partir do treinamento da rede neural por meio da exposição sucessiva de dados [PATTERSON; GIBSON, 2017].

A biblioteca para auxílio em aprendizagem de máquina Scikit-learn, é um módulo que integra uma vasta quantidade de algoritmos de machine learning de última geração para problemas supervisionados ou não. Em suma, Scikit-learning fornece um ambiente rico para implementações de algoritmos de aprendizagem de máquina, mantendo uma interface fácil para manipulação. Isso é reflexo da necessidade da análise de dados estatísticos por não especialistas nas indústrias de software, bem como nos campos externos à informática como a física e a engenharia [PEDREGOSA, 2011].

\subsubsection{Linguagem $C$ voltada para microcontroladores}

Por conseguir utilizar as funcionalidades do Assembly, que é uma linguagem de montagem que executa no hardware e possui um difícil entendimento, o C é utilizado em programação dos microcontroladores, que são placas que em termos gerais, podem ser definidos como processadores que foram encapsulados com memória, interface de entrada/saída de dados e dispositivos periféricos. Entre os periféricos estão conversores A/D (analógico/digital), temporizadores/contadores, interface para comunicação serial, watchdog programável, etc. Em outras palavras, são computadores comprimidos em um único recipiente. Tornaram-se comuns em diversos ramos da indústria a partir do final da década de 70 e atualmente existe um número crescente de opções disponíveis no mercado [BORGES, Geovany A. et al.,2008].

Com o tempo, a estrutura e arquitetura dos microcontroladores foram se aperfeiçoando e diversas empresas começaram a criar sua própria família de microcontroladores, aumentando sua disponibilidade e diminuindo gradativamente os custos. A popularidade desses produtos cresceu ainda mais nos últimos anos com sua considerável facilidade de acesso, gerando a possibilidade de desenvolvedores iniciantes adquirirem seu próprio microcontrolador para elaboração de pequenos ou complexos projetos.[BREVE \& BERNUY,2012].

\subsection{Aprendizagem Cooperativa}

Metodologias de ensino são as técnicas e abordagens utilizadas na transmissão e aquisição de conhecimento e se dividem em duas categorias essencialmente: metodologia tradicional e metodologias modernas. A metodologia tradicional corresponde ao método em que o professor é único detentor do conhecimento e o transmite ao aluno de maneira expositiva. Já as metodologias modernas são as técnicas que fogem do método tradicional de ensino. A aprendizagem cooperativa é uma metodologia moderna de ensino e está voltada para o protagonismo do aluno e o estudo em grupo [SOUSA, 2018].

O PACCE não visava somente melhorar o rendimento acadêmico e a aprendizagem diante de disciplinas mais complexas através do estudo em grupo, mas também buscando a interação e integração dos alunos.

Os cinco elementos bases da aprendizagem cooperativa, são: interdependência positiva, responsabilidade individual, interação face-a-face, habilidades sociais e processamento de grupo, sendo cada um deles essenciais para o funcionamento da metodologia [JOHNSON \& JOHNSON, 1999]. 
A interdependência positiva é uma característica em que os membros dependem um do outro para obterem êxito em seu objetivo. Os participantes possuem interesse não somente no seu próprio rendimento ao realizar uma tarefa, mas também no rendimento dos colegas. [BERNAL, 2000]. O alvo em comum possibilita o interesse do trabalho em grupo.

A responsabilidade individual ou autonomia relaciona - se com a interdependência positiva, de forma que apesar de haver interdependência entre os membros da célula de estudo, encontra - se também uma responsabilidade individual a qual cada elemento constituinte do grupo deve atender, de forma que além do grupo funcionar em conjunto, existam tarefas para cada um dos seus participantes individualmente [JOHNSON \& JOHNSON, 2008].

A interação face-a-face é essencial para a quebra do egocentrismo dos participantes de células de estudos [BERNAL, 2000]. Esta interação é caracterizada pelo desenvolvimento da união do grupo de modo que haja apoio e valorização do esforço de cada membro pelos outros [JOHNSON \& JOHNSON, 2000].

O sucesso do funcionamento e harmonia de um grupo dependem também de habilidades sociais. Essas habilidades do indivíduo, precisam ser moldadas para o convívio social. De modo que liderança, tomada de decisão, construção de confiança, comunicação e resolução de conflitos são habilidades que podem e devem ser desenvolvidas nos alunos [JOHNSON \& JOHNSON, 2000].

Por fim, o processamento de grupo é um meio de autoavaliação do grupo. Com ele o grupo analisa seu desenvolvimento, à medida que os objetivos são alcançados ou não, possibilitando, assim, haver identificação de condutas e comportamentos positivos e negativos que devem ser mantidos, modificados ou eliminados de acordo com as necessidades do grupo [JOHNSON \& JOHNSON, 1999].

\section{METODOLOGIA}

As células de programação faziam parte do Programa de Aprendizagem Cooperativa em Células Estudantis (PACCE), que era um programa de bolsas de monitoria que usava a metodologia da Aprendizagem Cooperativa e pertencia à Universidade Federal do Ceará.

A célula era a principal atividade do programa que tinha como objetivo a permanência dos alunos nos cursos, assim como a melhoria do rendimento acadêmico, a interação social e o desenvolvimento de habilidades sociais dos estudantes. $\mathrm{O}$ articulador de célula, líder e representante do grupo de estudo, passava por uma capacitação na metodologia de ensino no decorrer do ano.

$\mathrm{O}$ articulador estudava juntamente com os membros, a linguagem de programação e a Aprendizagem Cooperativa eram aplicadas através de dinâmicas e maratonas de programação que trabalhavam os cinco pilares da aprendizagem cooperativa.

Diferentes células de programação com duração de duas horas semanais eram implementadas dentro do PACCE, dentre elas, células voltadas para a linguagem Python, programação de aplicativos Android e, principalmente, linguagem C, que é a mais utilizada nos cursos de engenharia na UFC campus Sobral e é disciplina obrigatória do primeiro semestre dos cursos de engenharia da computação e engenharia elétrica do Campus Sobral. 
Para entender o impacto das células de programação, foi realizada uma pesquisa descritiva através de um questionário com alunos que participaram e alunos que não participaram de células de programação em que era aplicada a aprendizagem cooperativa.

A finalidade da pesquisa era saber como os alunos se saíam com a ajuda das células nas disciplinas de programação e, além disso, se os alunos dessas disciplinas conseguiam desenvolver suas habilidades sociais e pessoais.

Os alunos participantes da pesquisa são todos discentes da Universidade Federal do Ceará (UFC) campus Sobral. Destes, 97\% fazem parte do curso de engenharia da computação e $3 \%$ fazem parte do curso de engenharia elétrica. Além disso, a pesquisa tem caráter quantitativo e foram obtidas 41 respostas.

\section{RESULTADOS E DISCUSSÕES}

A pesquisa de caráter quantitativo foi realizada com alunos discentes da Universidade Federal do Ceará (UFC) campus Sobral, nas engenharias, ingressantes na disciplina de Programação Computacional. Destes, $97 \%$ fazem parte do curso de engenharia da computação e 3\% fazem parte do curso de engenharia elétrica. Ao todo foram obtidas 41 respostas.

Da amostra da pesquisa, 56,1\% não participaram de células de aprendizagem cooperativa e $43,9 \%$ participaram das células.

Segunda a pesquisa, a célula de programação auxiliava os alunos e ajudou os mesmos à obterem êxito na disciplina(Figura 1).

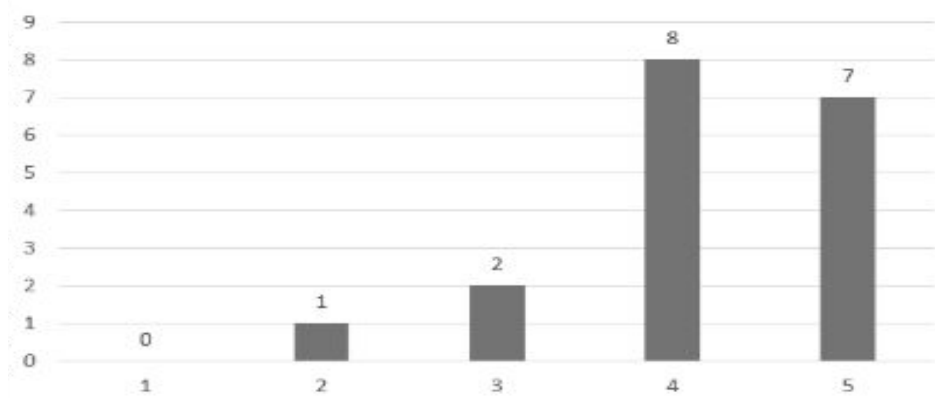

Figura 1. "A célula de aprendizagem cooperativa me ajudou a ter êxito na disciplina de Programação Computacional?".

Quando questionados se a célula ajudou no desenvolvimento de habilidades sociais as respostas foram positivas (Figura 2).

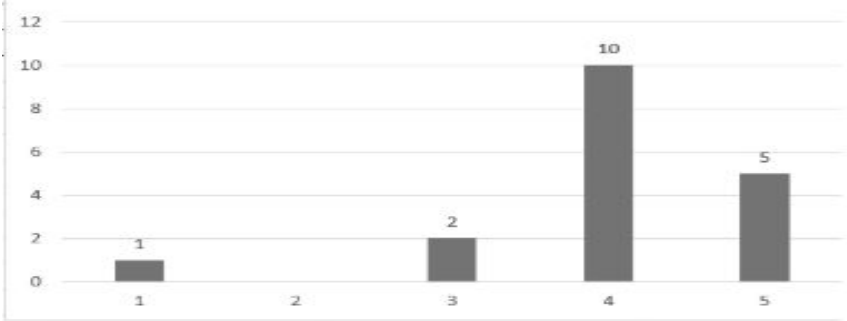

Figura 2: "A célula ajudou você a desenvolver habilidades como: trabalhar em grupo, autonomia e comunicação?" 
Os gráficos presentes nas figuras a seguir (Figura 3, 4, 5 e 6) comparam os níveis de domínio dos conteúdos de programação entre os alunos que participaram das células e os que não participaram.

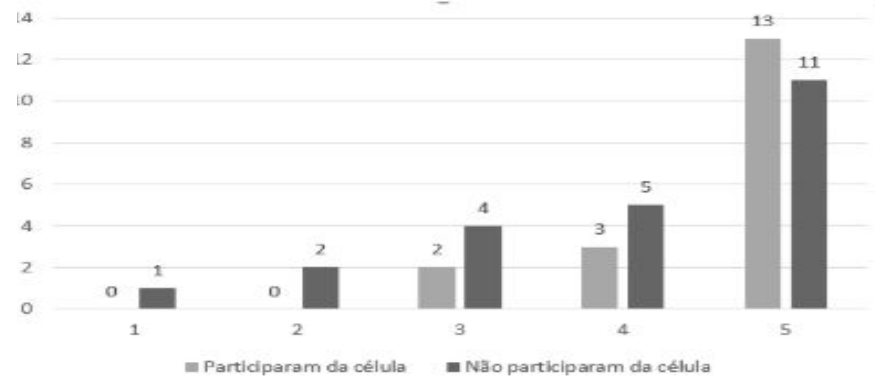

Figura 3. "Quanto você avalia seu domínio sobre a implementação de expressões condicionais como 'if-else-if'?"

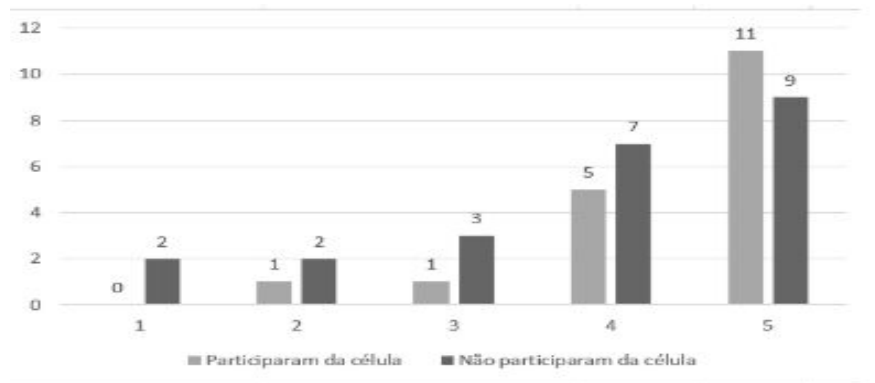

Figura 4. "Quanto você avalia seu domínio sobre as estruturas de repetição 'while' e 'for'?"

Referente aos conteúdos de estruturas condicionais e estruturas de repetição, que são conteúdos básicos e mais simples no estudo de linguagem de programação, os gráficos nas figuras acima apresentam o dado de que nenhum dos alunos que participaram das células de programação consideram seu domínio menor que mediano sobre estrutura condicional "if-else-if" e apenas 1 aluno (5\%) para estruturas de repetição "while" e "for", enquanto que $13 \%$ dos alunos (3) que não participaram das células de programação consideram seu domínio do conteúdo "if-else-if" abaixo da média e 17\% desses alunos (4) consideram seu domínio em estruturas de repetição abaixo da média.

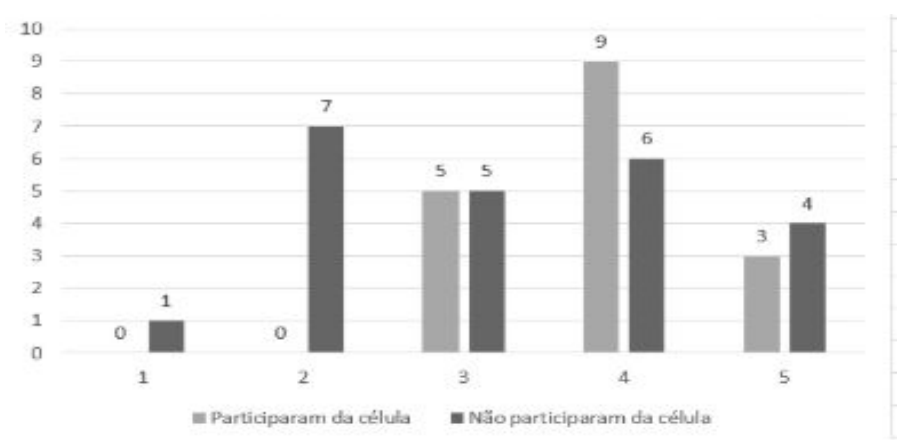

Figura 5. "Quanto você avalia seu domínio sobre a manipulação de vetores em linguagem de programação?" 


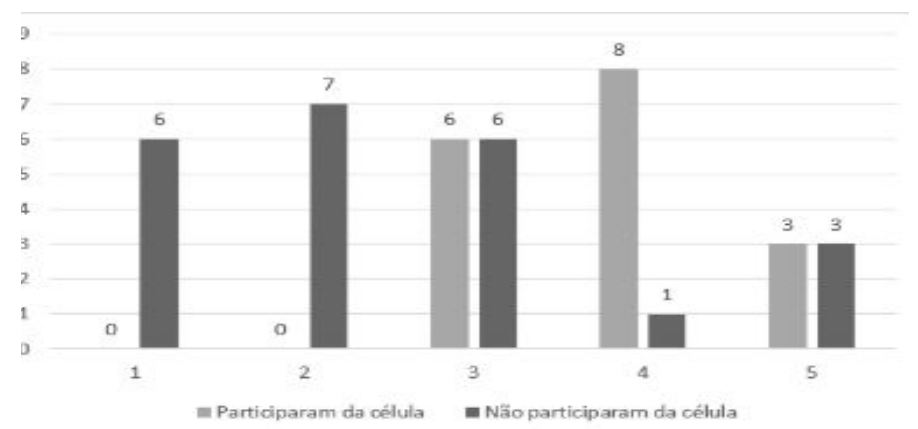

Figura 6. "Quanto você avalia seu domínio sobre a manipulação de ponteiros em linguagem de programação?"

Quando referente aos conteúdos "manipulação de vetores" e "manipulação de ponteiros" que são conteúdos mais complexos dentro do curso da disciplina de programação computacional para engenharia, a célula de programação alcança um destaque maior em comparação aos conteúdos mais básicos apresentados anteriormente.

Enquanto que nenhum dos alunos que participaram das células de programação consideram seu domínio desses conteúdos abaixo da média, dentre os alunos que não participaram da célula de programação 35\% considera seu domínio em "manipulação de vetores" abaixo da média. Em "manipulação de ponteiros" esse número aumenta para 56\%, são 13 alunos que consideram seu domínio sobre este conteúdo abaixo da média.

Referente ao domínio do conteúdo acima da média, considerando a média das quatro perguntas, $76 \%$ dos alunos que participaram da célula consideram seu domínio do conteúdo acima da média, em contraste com o valor de 50\% referente aos alunos que não participaram das células e consideram seu domínio dos conteúdos acima da média. Quando se refere somente às duas últimas questões, esses números se distanciam ainda mais. Enquanto 64\% dos alunos que participaram das células consideram seu domínio para estes conteúdos acima da média, apenas $30 \%$ dos alunos que não participaram da célula consideram seu domínio acima da média para os conteúdos mais complexos.

\section{CONSIDERAÇÕES FINAIS}

A aprendizagem cooperativa mostrou-se potencialmente útil como uma ferramenta na evolução dos estudantes de engenharia da UFC - Campus Sobral no aprendizado de linguagens de programação. Os resultados expressos nas pesquisas feitas, relataram que grande parte dos graduandos frequentadores das células tiveram êxito em adquirir conteúdos de lógica programacional e estrutura de dados. Utilizar técnicas e ferramentas de programação computacional é essencial para um profissional de engenharia. Logo, é perceptível observar que as células de programação podem desempenhar um papel importante nos cursos de engenharia, por que, aprofundam o domínio dos discentes sobre as diversas linguagens de programação, que podem ser utilizadas em ramos de pesquisa e execução como: Inteligência Artificial, Microcontroladores e Data Science.

\section{REFERÊNCIAS}

BERNAL, Ovejero et al. (2000) Aprendizaje Cooperativo: un eficaz instrumento de trabajo para las escuelas multiculturales y multiétnicas del siglo XXI. Revista Electrónica Iberoamericana de Psicología Social.

BORGES, Geovany A. et al. (2008) Desenvolvimento com microcontroladores Atmel AVR. 
BREVE, Matheus Montanini; BERNUY, Miguel Angel Chincaro. (2012) Introdução ao Desenvolvimento de Sistemas com Microcontroladores. ANAIS III Seminário de Pesquisa Jr (SepesqJr). Universidade Tecnológica Federal do Paraná. Disponível em: www. utfpr. edu. br/cornelioprocopio. Acesso em, v. 25.

HUNOLD, Murilo et al. (2014) Protótipo de prótese de mão robótica de lego controlada por sistema android para bi-amputado. In: XXIV Congresso Brasileiro de Engenharia Biomédica-CBEB.

JOHNSON, David W.; JOHNSON, Roger T.; HOLUBEC, Edythe Johnson. (1999) El aprendizaje cooperativo en el aula. Buenos Aires: Paidós.

JOHNSON, David; JOHNSON, Roger; SMITH, Karl. (2000) A aprendizagem cooperativa retorna às faculdades. Change, Jul/Aug98, v. 30, n. 4, p. p26.

JOHNSON, David W.; JOHNSON, Roger T. (2008) Cooperative learning. Blackwell Publishing Ltd.

KALISZYK, C.; CHOLLET, F.; SZEGEDY. C. (2017) Holstep: A machine learning dataset for higher-order logic theorem proving. In: International Conference on Learning Representations (ICLR).

LECHETA, R. R. (2015) Google Android 4a edição. Novatec Editora.

OLIVEIRA, I.; BALBINOT, A. (2014) Eletrocardiograma Portátil Através De Aplicativo Para Sistema Operacional Android. In: XXIV-Congresso Nacional de Engenharia Biomédica. Uberlândia.

PATTERSON, J.; GIBSON, A. (2017) Deep Learning: A Practitioner's Approach. Beijing: O'Reilly. ISBN 978-1-4919-1425-0. Dispon 'ivel em: https://www.safaribooksonline.com/library/view/deep-learning/9781491924570/i.

PEDREGOSA, F., et. al. (2011) Scikit-learn: Machine learning in Python. Journal of Machine Learning Research, 12:2825-2830.

RODRIGUES, A. A. et al. (2014) Desenvolvimento de módulo controlador para sistemas de irrigação com interface android-bluetooth. XLIII CONGRESSO BRASILEIRO DE ENGENHARIA AGRÍCOLA. Campo Grande.

SOUSA, Francisco Thales Rocha et al. (2018) Metodologia de ensino moderna orientada a projetos: modelo colaborativo de aprendizagem aplicado à disciplina de Eletrônica Digital. Revista de Informática Aplicada, v. 14, n. 1. 